\title{
Operational Semantics Using the Partiality Monad
}

\author{
Nils Anders Danielsson \\ Chalmers University of Technology and University of Gothenburg \\ nad@chalmers.se
}

\begin{abstract}
The operational semantics of a partial, functional language is often given as a relation rather than as a function. The latter approach is arguably more natural: if the language is functional, why not take advantage of this when defining the semantics? One can immediately see that a functional semantics is deterministic and, in a constructive setting, computable.

This paper shows how one can use the coinductive partiality monad to define big-step or small-step operational semantics for lambda-calculi and virtual machines as total, computable functions (total definitional interpreters). To demonstrate that the resulting semantics are useful type soundness and compiler correctness results are also proved. The results have been implemented and checked using Agda, a dependently typed programming language and proof assistant.
\end{abstract}

Categories and Subject Descriptors F.3.2 [Logics and Meanings of Programs]: Semantics of Programming Languages-Operational semantics; D.1.1 [Programming Techniques]: Applicative (Functional) Programming; E.1 [Data Structures]; F.3.1 [Logics and Meanings of Programs]: Specifying and Verifying and Reasoning about Programs-Mechanical verification

Keywords Dependent types; mixed induction and coinduction; partiality monad

\section{Introduction}

Consider the untyped $\lambda$-calculus with a countably infinite set of constants $c$ :

$$
t::=c|x| \lambda x . t \mid t_{1} t_{2}
$$

Closed terms written in this language can compute to a value (a constant $c$ or a closure $\lambda x . t \rho$ ), but they can also go wrong (crash) or fail to terminate.

How would you write down an operational semantics for this language? A common choice is to define the semantics as an inductively defined relation, either using small steps or big steps. For an example of the latter, see Figure 1: $\rho \vdash t \Downarrow v$ means that the term $t$ can terminate with the value $v$ when evaluated in the environment $\rho$. However, as noted by Leroy and Grall (2009), this definition provides no way to distinguish terms which go wrong from terms which fail to terminate. If we want to do this, then we can define two more relations, see Figure 2: $\rho \vdash t \Uparrow$, defined coinductively,

(c) ACM, 2012. This is the author's version of the work. It is posted here by permission of ACM for your personal use. Not for redistribution. The definitive version was published in the Proceedings of the 17th ACM SIGPLAN international conference on Functional programming (2012), http://doi.acm.org/10.1145/ 2364527.2364546 .

$$
\begin{array}{cll}
\rho \vdash c \Downarrow c & \frac{\rho(x)=v}{\rho \vdash x \Downarrow v} \quad \rho \vdash \lambda x . t \Downarrow \lambda x . t \rho \\
\frac{\rho \vdash t_{1} \Downarrow \lambda x . t^{\prime} \rho^{\prime}}{\rho \vdash t_{2} \Downarrow v^{\prime}} \quad \rho^{\prime}, x=v^{\prime} \vdash t^{\prime} \Downarrow v \\
\rho \vdash t_{1} t_{2} \Downarrow v
\end{array}
$$

Figure 1. A call-by-value operational semantics for the untyped $\lambda$ calculus with constants, specifying which terms can terminate with what values (very close to a semantics given by Leroy and Grall (2009)).

$$
\begin{aligned}
& \frac{\rho \vdash t_{1} \Uparrow}{\rho-t_{1} t_{2} \Uparrow} \quad \frac{\rho \vdash t_{1} \Downarrow v \quad \rho \vdash t_{2} \Uparrow}{\rho \vdash t_{1} t_{2} \Uparrow} \\
& \frac{\rho \vdash t_{1} \Downarrow \lambda x . t^{\prime} \rho^{\prime} \quad \rho \vdash t_{2} \Downarrow v^{\prime} \quad \rho^{\prime}, x=v^{\prime} \vdash t^{\prime} \Uparrow}{\rho \vdash t_{1} t_{2} \Uparrow} \\
& \rho \vdash t \downarrow \stackrel{\text { def }}{=} \neg(\exists v \cdot \rho \vdash t \Downarrow v) \wedge \neg(\rho \vdash t \Uparrow)
\end{aligned}
$$

Figure 2. Two more operational semantics for the untyped $\lambda$ calculus with constants, specifying which terms can fail to terminate or go wrong. The definition written using double lines is coinductive, and is taken almost verbatim from Leroy and Grall (2009).

means that the term $t$ can fail to terminate when evaluated in the environment $\rho$; and $\rho \vdash t$ 々 means that $t$ goes wrong.

Now we have a complete definition. However, this definition is somewhat problematic:

1. There are four separate rules which refer to application. For a small language this may be acceptable, but for large languages it seems to be easy to forget some rule, and "rule duplication" can be error-prone.

2. It is not immediately obvious whether the semantics is deterministic and/or computable: these properties need to be proved.

3. If we want to define an interpreter which is correct by construction, then the setup with three relations is awkward. Consider the following type-signature, where $\uplus_{-}$is the sum type constructor:

$$
\text { eval : } \forall \rho t \rightarrow(\exists v . \rho \vdash t \Downarrow v) \uplus \rho \vdash t \Uparrow \uplus \rho \vdash t \text { 々 }
$$

This signature states that, for any environment $\rho$ and term $t$, the interpreter either returns a value $v$ and a proof that $t$ can terminate with this value when evaluated in the given environment; or a proof that $t$ can fail to terminate; or a proof that $t$ goes wrong. It should be clear that it is impossible to implement eval 
in a total, constructive language, as this amounts to solving the halting problem.

The situation may have been a bit less problematic if we had defined a small-step semantics instead, but small-step semantics are not necessarily better: Leroy and Grall (2009) claim that "big-step semantics is more convenient than small-step semantics for some applications", including proving that a compiler is correct.

I suggest another approach: define the semantics as a function in a total meta-language, using the partiality monad (Capretta 2005) to represent non-termination, where the partiality monad is defined coinductively as $A_{\perp}=\nu X . A \uplus X$. If this approach is followed then we avoid all the problems above:

1. We have one clause for applications, and the meta-language is total, so we cannot forget a clause.

2. The semantics is a total function, and hence deterministic and computable.

3. The semantics is an interpreter, and its type signature does not imply that we solve the halting problem:

$$
\llbracket-\rrbracket: \text { Term } \rightarrow \text { Environment } \rightarrow(\text { Maybe Value })_{\perp}
$$

An additional advantage of using a definitional interpreter is that this can make it easy to test the semantics (if the interpreter is not too inefficient). Such tests can be useful in the design of non-trivial languages (Aydemir et al. 2005).

The main technical contribution of this paper is that I show that one can prove typical meta-theoretical properties directly for a semantics defined using the partiality monad:

- A big-step, functional semantics is defined and proved to be classically equivalent to the relational semantics above (Sections 3 and 5; for simplicity well-scoped de Bruijn indices are used instead of names).

- Type soundness is proved for a simple type system with recursive types (Section 4).

- The meaning of a virtual machine is defined as a small-step, functional semantics (Section 6).

- A compiler correctness result is proved (Section 7).

- The language and the type soundness and compiler correctness results are extended to a non-deterministic setting in order to illustrate that the approach can handle languages where some details-like evaluation order-are left up to the compiler writer (Section 8).

- Finally Section 9 contains a brief discussion of term equivalences (applicative bisimilarity and contextual equivalence).

As far as I know these are the first proofs of type soundness or compiler correctness for operational semantics defined using the partiality monad. The big-step semantics avoids the rule duplication mentioned above, and this is reflected in the proofs: there is only one case for application, as opposed to four cases in some corresponding proofs for relational semantics due to Leroy and Grall (2009). Related work is discussed further in Section 1.3.

\subsection{Operational?}

At this point some readers may complain that $\llbracket-\rrbracket$ does not define an operational semantics, but rather a denotational one. Perhaps a better term would be "hybrid operational/denotational", but the semantics is not denotational:

- It is not defined in a compositional way: $\llbracket t \rrbracket$ is not defined by recursion on the structure of $t$, but rather a combination of corecursion and structural recursion (see Section 3).
- Furthermore the "semantic domain" is rather syntactic: it includes closures, and is not defined as the solution to a domain equation.

I do not see this kind of semantics as an alternative to denotational semantics, but rather as an alternative to usual operational ones. (See also the discussion of term equivalences in Section 9.)

\subsection{Mechanisation}

The development presented below has been formalised in the dependently typed, functional language Agda (Norell 2007; Agda Team 2012), and the code has been made available to download.

In order to give a clear picture of how the results can be mechanised Agda-like code is also used in the paper. Unfortunately Agda's support for total corecursion is somewhat limited, ${ }^{1}$ so to avoid distracting details the code is written in an imaginary variant of Agda with a very clever productivity checker (and some other smaller changes). The accompanying code is written in actual Agda, sometimes using workarounds (Danielsson 2010) to convince Agda that the code is productive. There are also other, minor differences between the accompanying code and the code in the paper.

\subsection{Related Work}

Reynolds (1972) discusses definitional interpreters, and there is a large body of work on using monads to structure semantics and interpreters, going back at least to Moggi (1991) and Wadler (1992).

The toy language above is taken from Leroy and Grall (2009), who bring up some of the disadvantages of (inductive) big-step semantics mentioned above. The type system in Section 4 is also taken from Leroy and Grall, who discuss various formulations of type soundness (but not the main formulations given below). Finally the virtual machine and compiler defined in Sections 6-7 are also taken from Leroy and Grall, who give a compiler correctness proof.

Leroy and Grall also define a semantics based on approximations: First the semantics is defined (functionally) at "recursion depth" $\mathrm{n}$; if $n=0$, then the result $\perp$ is returned. This function is similar to the functional semantics $\llbracket-\rrbracket$ defined in Section 3, but defined using recursion on $n$ instead of corecursion and the partiality monad. The semantics of a term $t$ is then defined (relationally) to be $s$ if there is a recursion depth $n_{0}$ such that the semantics at recursion depth $n$ is $s$ for all $n \geqslant n_{0}$. Leroy and Grall prove that this semantics is equivalent to a relational, big-step semantics. This proof is close to the proof in Section 5 which shows that $\llbracket-\rrbracket$ is equivalent to a relational, big-step semantics.

Further comparisons to the work of Leroy and Grall is included below.

The type soundness proof in Section 4 is close to proofs given by Tofte (1990) and Milner and Tofte (1991). They use ordinary, inductive big-step definitions to give semantics of languages with cyclic closures, define typing relations for values coinductively (as greatest fixpoints of monotone operators $F$ ), and use coinduction $(x \in v F$ if $x \in X$ for some $X \subseteq F(X))$ to prove that certain values have certain types. In this paper the value typing relation is defined inductively rather than coinductively. However, another typing relation, that for possibly non-terminating computations, is defined coinductively, and the proof still uses coinduction (which takes the form of corecursion, see Section 2).

Capretta (2005) discusses the partiality monad, and gives a semantics for partial recursive functions (primitive recursive functions plus minimisation) as a function of type $\forall n$. $\left(\mathbb{N}^{n} \rightarrow \mathbb{N}\right) \rightarrow$ $\left(\mathbb{N}_{\perp}^{n} \rightarrow \mathbb{N}_{\perp}\right)$.

\footnotetext{
${ }^{1}$ The same applies to Coq (Coq Development Team 2011).
} 
Nakata and Uustalu (2009) define coinductive big-step and small-step semantics, in both relational and functional style, for a while language. Their definitions do not use the partiality monad, but are trace-based, and have the property that the trace can be computed (productively) for any source term, converging or diverging. My opinion is that the relational big-step definition is rather technical and brittle; the authors discuss several modifications to the design which lead to absurd results, like while true do skip having an arbitrary trace. The functional big-step semantics avoids these issues, because the semantics is required to be a productive function from a term and an initial state to a trace. Nakata and Uustalu have extended their work to a while language with interactive input/output (2010), but in this work they use relational definitions.

Paulin-Mohring (2009) defines partial streams using (essentially) the partiality monad, shows that partial streams form a pointed CPO, and uses this CPO to define a functional semantics for (a minor variation of) Kahn networks.

Benton et al. (2009) use the partiality monad to construct a lifting operator for CPOs, and use this operator to give denotational semantics for one typed and one untyped $\lambda$-calculus; the former semantics is crash-free by construction, the latter uses $\perp$ to represent crashes. Benton and Hur (2009) define a compiler from one of these languages to a variant of the SECD machine (with a relational, small-step semantics), and prove compiler correctness.

Ghani and Uustalu (2004) introduce the partiality monad transformer, $\lambda M A$ A. $v X . M(A \uplus X)$. (In the setting of Agda $M$ should be restricted to be strictly positive.)

Goncharov and Schröder (2011) use the partiality monad transformer (they use the term resumption monad transformer) to give a class of functional semantics for a concurrent language.

Rutten (1999) defines an operational semantics for a while language corecursively as a function, using a "non-constructive" variant of the partiality monad, $A_{\perp}=(A \times \mathbb{N}) \uplus\{\infty\}$ (where $\infty$ represents non-termination and the natural number stands for the number of computation steps needed to compute the value of type $A$ ). With this variant of the monad the semantics is not a computable function, because the semantics returns $\infty$ iff a program fails to terminate. Rutten also discusses weak bisimilarity and explains how to construct a compositional semantics from the operational one.

Cousot and Cousot $(1992,2009)$ describe bi-inductive definitions, which generalise inductive and coinductive definitions, and give a number of examples of their use. One of their examples is a big-step semantics for a call-by-value $\lambda$-calculus. This semantics captures both terminating and non-terminating behaviours in a single definition, with less "duplication" of rules than in Figures 1-2, but more than in Section 3. An operator $F$ on $\wp($ Term $\times($ Term $\cup\{\perp\}))$, where Term stands for the set of terms and $\perp$ stands for non-termination, is first defined by the following inference rules (where $v$ ranges over values):

$$
\begin{gathered}
v \Rightarrow v \quad \frac{t_{1} \Rightarrow \perp}{t_{1} t_{2} \Rightarrow \perp} \quad \frac{t_{1} \Rightarrow v \quad t_{2} \Rightarrow \perp}{t_{1} t_{2} \Rightarrow \perp} \\
\frac{t_{1} \Rightarrow \lambda x . t \quad t_{2} \Rightarrow v \quad t[x:=v] \Rightarrow r}{t_{1} t_{2} \Rightarrow r}
\end{gathered}
$$

These rules should neither be read inductively nor coinductively. The semantics is instead obtained as the least fixpoint of $F$ with respect to the order _ᄃ_ defined by

$$
X \sqsubseteq Y=X^{+} \subseteq Y^{+} \wedge X^{-} \supseteq Y^{-},
$$

where $Z^{+}=\{(t, s) \in Z \mid s \neq \perp\}$ and $Z^{-}=Z \backslash Z^{+} . F$ is not monotone with respect to $\_-{ }_{-}$(which forms a complete lattice), so Cousot and Cousot give an explicit proof of the existence of a least fixpoint (for a closely related semantics).

\section{The Partiality Monad}

Agda is a total language (assuming that the implementation is bugfree, etc.). Ordinary data types are inductive. For instance, we can define the type Fin $n$ of natural numbers less than $n$, and the type $V e c A n$ of $A$-lists of length $n$, as follows:

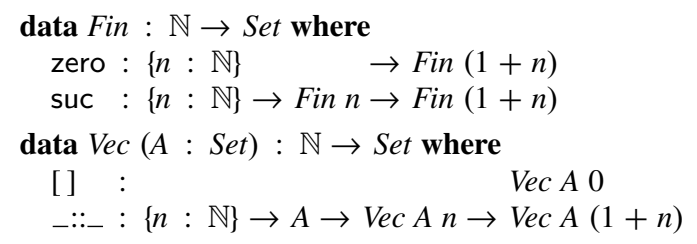

(Cons is an infix operator, _::_; the underscores mark the argument positions.) Inductive types can be destructed using structural recursion. As an example we can define a safe lookup/indexing function:

$$
\begin{aligned}
& \text { lookup : }\{A: \text { Set }\}\{n: \mathbb{N}\} \rightarrow \text { Fin } n \rightarrow \operatorname{Vec} A n \rightarrow A \\
& \text { lookup zero }(x:: x s)=x \\
& \text { lookup (suc } i)(x:: x s)=\text { lookup } i x s
\end{aligned}
$$

The arguments within braces, $\{\ldots\}$, are implicit, and can be omitted if Agda can infer them. To avoid clutter most implicit argument declarations are omitted, together with a few explicit instantiations of implicit arguments.

Agda also supports "infinite" data through the use of coinduction (Coquand 1994). Coinductive types can be introduced using suspensions: $\infty A$ is the type of suspensions, that if forced give us something of type $A$. Suspensions can be forced using ${ }^{b}$, and created using $\sharp_{-}$:

$$
\begin{array}{r}
b^{\prime}: \infty A \rightarrow \quad A \\
\sharp_{-}: \quad A \rightarrow \infty A
\end{array}
$$

(Here $\#_{-}$is a tightly binding prefix operator. In this paper nothing binds tighter except for ordinary function application.)

The partiality monad is defined coinductively as follows:

$$
\begin{gathered}
\text { data }_{-\perp}(A: \text { Set }): \text { Set } \text { where } \\
\text { now : } A \quad \rightarrow A_{\perp} \\
\text { later }: \infty\left(A_{\perp}\right) \rightarrow A_{\perp}
\end{gathered}
$$

You can read this as the greatest fixpoint $\nu X . A \uplus X .^{2}$ The constructor now returns a value immediately, and later postpones a computation. Computations can be postponed forever:

$$
\begin{aligned}
& \text { never }: A_{\perp} \\
& \text { never }=\text { later }(\sharp \text { never })
\end{aligned}
$$

Here never is defined using corecursion, in a productive way: even though never can unfold forever, the next constructor can always be computed in a finite number of steps. Note that structural recursion is not supported for coinductive types, as this would allow the definition of non-productive functions.

The partiality monad is a monad, with now as its return operation, and bind defined corecursively as follows:

$$
\begin{aligned}
& \varlimsup_{-}: A_{\perp} \rightarrow\left(A \rightarrow B_{\perp}\right) \rightarrow B_{\perp} \\
& \text { now } x \gg f=f x \\
& \text { later } x \gg f=\operatorname{later}\left(\sharp\left({ }^{b} x \gg f\right)\right)
\end{aligned}
$$

If $x$ fails to terminate, then $x \gg f$ also fails to terminate, and if $x$ terminates with a value, then $f$ is applied to that value.

It is easy to prove the monad laws up to (strong) bisimilarity, which is a coinductively defined relation:

\footnotetext{
2 This is not entirely correct in the current version of Agda (Altenkirch and Danielsson 2010), but for the purposes of this paper the differences are irrelevant.
} 


$$
\begin{aligned}
& \text { data } \_\cong A_{\perp} \rightarrow A_{\perp} \rightarrow \text { Set } \text { where } \\
& \text { now : } \\
& \text { later : } \infty\left(^{b} x \cong{ }^{b} y\right) \rightarrow \text { later } x \cong \text { now } x
\end{aligned}
$$

(Note that the constructors have been overloaded.) This equivalence relation relates diverging computations, and it also relates computations which converge to the same value using the same number of steps.

Note that $\cong \cong_{-}$is a type of potentially infinite proof terms. Proving $x \cong y$ amounts to constructing a term with this type. This proof technique is quite different from the usual coinductive proof technique (where $x \in v F$ for a monotone $F$ if $x \in X$ for some $X \subseteq F(X)$ ), so let me show in detail how one can prove that bind is associative:

associative :

$$
\left(x: A_{\perp}\right)\left(f: A \rightarrow B_{\perp}\right)\left(g: B \rightarrow C_{\perp}\right) \rightarrow
$$$$
(x \gg=f \gg g) \cong(x \gg \lambda y \rightarrow f y \gg=g)
$$

We can do this using corecursion and case analysis on $x$ :

$$
\text { associative (now } x \text { ) } f g=\text { ? }
$$$$
\text { associative (later } x \text { ) } f g=\text { ? }
$$

We can ask Agda what types the two goals (?) have. The first one has type $f x \gg g \cong f x \gg g$, and can be completed by appeal to reflexivity $\left(\right.$ refl- $\cong:\left(x: A_{\perp}\right) \rightarrow x \cong x$ can be proved separately):

$$
\text { associative (now } x) f g=r e f l \cong(f x \gg g)
$$

The second goal has type later $s_{1} \cong$ later $s_{2}$ for some suspensions $s_{1}$ and $s_{2}$, so we can refine the goal using a later constructor and a suspension:

$$
\text { associative (later } x) f g=\text { later }(\sharp \text { ?) }
$$

The new goal has type

$$
\left({ }^{b} x \gg f \gg g\right) \cong\left({ }^{b} x \gg \lambda y \rightarrow f y \gg g\right),
$$

so we can conclude by appeal to the coinductive hypothesis:

$$
\text { associative (later } x) f g=\text { later }\left(\sharp \text { associative }\left({ }^{b} x\right) f g\right. \text { ) }
$$

Note that the proof is productive. Agda can see this, because the corecursive call is guarded by a constructor and a suspension.

Strong bisimilarity is very strict. In many cases weak bisimilarity, which ignores finite differences in the number of steps, is more appropriate: ${ }^{3}$

$$
\begin{aligned}
& \text { data } \approx_{-}: A_{\perp} \rightarrow A_{\perp} \rightarrow \text { Set where } \\
& \text { now : } \quad \text { now } x \approx \text { now } x \\
& \text { later : } \infty\left({ }^{b} x \approx{ }^{b} y\right) \rightarrow \text { later } x \approx \text { later } y \\
& \text { later }{ }^{1}: \quad b^{b} \approx y \rightarrow \text { later } x \approx y \\
& \text { later }^{r}: \quad x \approx{ }^{b} y \rightarrow \quad x \approx \text { later } y
\end{aligned}
$$

This relation is defined using mixed induction and coinduction (induction nested inside coinduction, $\nu X . \mu Y . F X Y$ ). Note that later is coinductive, while later ${ }^{1}$ and later ${ }^{r}$ are inductive. An infinite sequence of later constructors is allowed, for instance to prove never $\approx$ never:

$$
\begin{aligned}
& \text { allowed }: \text { never } \approx \text { never } \\
& \text { allowed }=\text { later }(\sharp \text { allowed })
\end{aligned}
$$

However, only a finite number of consecutive later ${ }^{l}$ and later ${ }^{r}$ constructors is allowed, because otherwise we could prove never $\approx$ now $x$ :

\footnotetext{
${ }^{3}$ Capretta (2005) defines weak bisimilarity in a different but equivalent way.
}

$$
\begin{aligned}
& \text { disallowed }: \text { never } \approx \text { now } x \\
& \text { disallowed }=\text { later }^{1} \text { disallowed }
\end{aligned}
$$

On the other hand, because the induction is nested inside the coinduction it is fine to use an infinite number of later ${ }^{1}$ or later ${ }^{\mathrm{r}}$ constructors if they are non-consecutive, with intervening later constructors:

$$
\begin{aligned}
& \text { also-allowed }: \text { never } \approx \text { never } \\
& \text { also-allowed }=\text { later }^{\mathrm{r}}(\text { later }(\sharp \text { also-allowed }))
\end{aligned}
$$

If we omit the later ${ }^{\mathrm{r}}$ constructor from the definition of weak bisimilarity, then we get a preorder _ $z_{-}$with the property that $x \gtrsim y$ holds if $y$ terminates in fewer steps than $x$ (with the same value), but not if $x$ terminates in strictly fewer steps than $y$, or if one of the two computations terminates and the other does not:

$$
\begin{aligned}
& \text { data }_{-} \gtrsim_{-}: A_{\perp} \rightarrow A_{\perp} \rightarrow \text { Set } \text { where } \\
& \text { now }: \text { now } x \gtrsim \text { now } x \\
& \text { later }: \infty\left({ }^{b} x \gtrsim b y\right) \rightarrow \text { later } x \gtrsim \text { later } y \\
& \text { later }^{1}: \quad b^{b} x \geqslant y \rightarrow \text { later } x \gtrsim \quad y
\end{aligned}
$$

It is easy to prove that $x \cong y$ implies $x \gtrsim y$, which in turn implies $x \approx y$.

The three relations above are transitive, but one needs to be careful when using transitivity in corecursive proofs, because otherwise one can "prove" absurd things. For instance, given refl- $\approx$ : $\left(x: A_{\perp}\right) \rightarrow x \approx x$ and trans $\approx: x \approx y \rightarrow y \approx z \rightarrow x \approx z$ we can "prove" that weak bisimilarity is trivial:

$$
\begin{aligned}
\text { trivial }:\left(x y: A_{\perp}\right) \rightarrow x \approx y \\
\text { trivial } x y= \\
\text { trans }-\approx\left(\text { later }^{\mathrm{r}}(\text { refl- } \approx x)\right) \\
\quad\left(\text { trans }-\approx^{-}\left(\text {later }^{\sharp} \text { trivial } x y\right)\right) \\
\left.\quad\left(\text { later }^{1}(\text { refl- } \approx y)\right)\right)
\end{aligned}
$$

This "proof" uses the following equational reasoning steps: $x \approx$ later $(\sharp x) \approx \operatorname{later}(\sharp y) \approx y$. The problem is that trivial is not productive: trans- $\approx$ is "too strict". This issue is closely related to the problem of weak bisimulation up to weak bisimilarity (Sangiorgi and Milner 1992).

Fortunately some uses of transitivity are safe. For instance, if we are proving a weak bisimilarity, then it is safe to make use of already proved greater-than results, in the following way (where $y \lesssim z$ is a synonym for $z \gtrsim y$ ):

$$
\begin{aligned}
& x \gtrsim y \rightarrow y \approx z \rightarrow x \approx z \\
& x \approx y \rightarrow y \lesssim z \rightarrow x \approx z
\end{aligned}
$$

(Compare Sangiorgi and Milner's "expansion up to $\lesssim$ ") Agda does not provide a simple way to show that these lemmas are safe, but this could be done using sized types as implemented in MiniAgda (Abel 2010). ${ }^{4}$ With sized types one can define $x \approx^{i} y$ to stand for potentially incomplete proofs of $x \approx y$ of size (at least) $i$, and prove the following lemma:

$$
\forall i . x \gtrsim y \rightarrow y \approx^{i} z \rightarrow x \approx^{i} z
$$

This lemma is not "too strict": the type tells us that the (bound on the) size of the incomplete definition is preserved. Unfortunately MiniAgda, which is a research prototype, is very awkward to use in larger developments.

For more details about coinduction and corecursion in Agda, and further discussion of transitivity in a coinductive setting, see Danielsson and Altenkirch (2010).

\footnotetext{
${ }^{4}$ The experimental implementation of sized types in Agda does not support coinduction.
} 


\section{A Functional, Operational Semantics}

This section defines an operational semantics for the untyped $\lambda$ calculus with constants. Let us start by defining the syntax of the language. Just as Leroy and Grall (2009) I use de Bruijn indices to represent variables, but I use a "well-scoped" approach, using the type system to keep track of the free variables. Terms of type Tm $n$ have at most $n$ free variables:

$$
\begin{aligned}
& \text { data } \operatorname{Tm}(n: \mathbb{N}): \text { Set where } \\
& \text { con : } \mathbb{N} \quad \rightarrow \text { Tm } n \quad \text {-- Constant. } \\
& \text { var : Fin } n \rightarrow T m n-- \text { Variable. } \\
& \text { lam : } \operatorname{Tm}(1+n) \rightarrow T m n-\text { Abstraction. } \\
& \text { _-_ : } \operatorname{Tm} n \rightarrow \operatorname{Tm} n \rightarrow \operatorname{Tm} n \quad \text {-- Application. }
\end{aligned}
$$

Environments and values are defined mutually:

$$
\begin{aligned}
& \text { mutual } \\
& \text { Env : } \mathbb{N} \rightarrow \text { Set } \\
& \text { Env } n=\text { Vec Value } n \\
& \text { data Value : Set where } \\
& \quad \text { con : } \mathbb{N} \\
& \quad \text { lam }: \operatorname{Tm}(1+n) \rightarrow \text { Env } n \rightarrow \text { Value -- Constant. }
\end{aligned}
$$

Note that the body of a closure has at most one free variable which is not bound in the environment.

The language supports two kinds of "effects", partiality and crashes. The partiality monad is used to represent partiality, and the maybe monad is used to represent crashes:

$$
\llbracket-\rrbracket: \operatorname{Tm} n \rightarrow \text { Env } n \rightarrow(\text { Maybe Value })_{\perp}
$$

(Maybe $A$ has two constructors, nothing : Maybe $A$ and just : $A \rightarrow$ Maybe A.) The combined monad is the maybe monad transformer ( $\lambda M A$ A. $M$ (Maybe A)) applied to the partiality monad. We can define a failing computation, as well as return and bind, as follows:

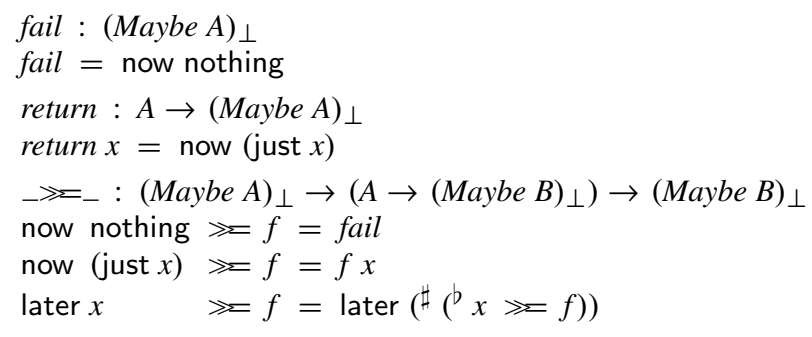

It should also be possible to use the reader monad transformer to handle the environment, but I believe that this would make the code harder to follow.

With the monad in place it is easy to define the semantics using two mutually (co)recursive functions:

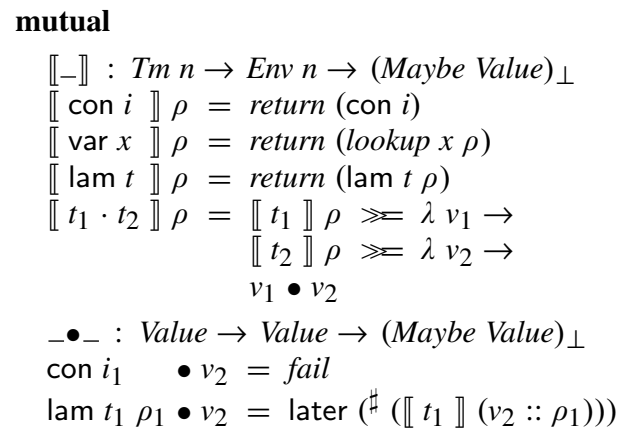

Constants are returned immediately, variables are looked up in the environment, and abstractions are paired up with the environment to form a closure. The interesting case is application: $t_{1} \cdot t_{2}$ is evaluated by first evaluating $t_{1}$ to a value $v_{1}$, then (if the evaluation of $t_{1}$ terminates without a crash) $t_{2}$ to $v_{2}$, and finally evaluating the application $v_{1} \bullet v_{2}$. If $v_{1}$ is a constant, then we crash. If $v_{1}$ is a closure, then a later constructor is emitted and the closure's body is evaluated in its environment extended by $v_{2}$. The result contains one later constructor for every $\beta$-redex that has been reduced (infinitely many in case of non-termination).

Note that this is a call-by-value semantics, with functions evaluated before arguments. Note also that the semantics is not compositional, i.e. not defined by recursion on the structure of the term, so it is not a denotational semantics. (It would be if _ •

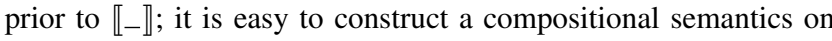
top of this one.)

Agda does not accept the code above; it is not obvious to the productivity checker that $\llbracket-\rrbracket$ and _- $\bullet_{-}$are total (productive) functions. If bind had been a constructor, then Agda would have found that the code uses a lexicographic combination of guarded corecursion and structural recursion: every call path from 【-】 to $\llbracket-\rrbracket$ is either

1. guarded by one or more constructors and at least one suspension (and nothing else), or

2. guardedness is "preserved" (zero or more constructors/suspensions), and the term argument becomes strictly smaller.

Now, bind is not a constructor, but it does preserve guardedness: it takes apart its first argument, but introduces a new suspension before forcing an old one-in MiniAgda one can show that bind preserves the sizes of its arguments. For a formal explanation of totality, see the accompanying code. ${ }^{5}$

The semantics could also have been defined using continuationpassing style, and then we could have avoided the use of bind:

$$
\begin{aligned}
& \text { mutual } \\
& \llbracket-\rrbracket_{\mathrm{CPS}}: \text { Tm } n \rightarrow \text { Env } n \rightarrow\left(\text { Value } \rightarrow(\text { Maybe } A)_{\perp}\right) \rightarrow \\
& \text { (Maybe A) } \perp \\
& \llbracket \operatorname{con} i \rrbracket_{\mathrm{CPS}} \rho k=k(\operatorname{con} i) \\
& \llbracket \operatorname{var} x \rrbracket_{\mathrm{CPS}} \rho k=k(\text { lookup } x \rho) \\
& \llbracket \operatorname{lam} t \rrbracket_{\mathrm{CPS}} \rho k=k(\operatorname{lam} t \rho) \\
& \llbracket t_{1} \cdot t_{2} \rrbracket_{\mathrm{CPS}} \rho k=\llbracket t_{1} \rrbracket_{\mathrm{CPS}} \rho\left(\lambda v_{1} \rightarrow\right. \\
& \llbracket t_{2} \rrbracket_{\mathrm{CPS}} \rho\left(\lambda v_{2} \rightarrow\right. \\
& \left.\left.\left(v_{1} \bullet_{\mathrm{CPS}} v_{2}\right) k\right)\right) \\
& { }^{-}{ }_{\mathrm{CPS}}-: \text { Value } \rightarrow \text { Value } \rightarrow\left(\text { Value } \rightarrow(\text { Maybe } A)_{\perp}\right) \rightarrow \\
& \text { (Maybe A) } \perp \\
& \left(\begin{array}{lll}
\text { con } i_{1} & \bullet \text { CPS } & v_{2}
\end{array}\right) k=\text { fail } \\
& \left(\operatorname{lam} t_{1} \rho_{1} \bullet_{\mathrm{CPS}} v_{2}\right) k=\operatorname{later}\left(\sharp\left(\llbracket t_{1} \rrbracket_{\mathrm{CPS}}\left(v_{2}:: \rho_{1}\right) k\right)\right)
\end{aligned}
$$

This definition would not have made the productivity checker any happier (it is productive, though, see the accompanying code). However, it avoids the inefficient implementation of bind; note that bind traverses the full prefix of later constructors before encountering the now constructor, if any.

Before we leave this section, let us work out a small example. The term $(\lambda x . x x)(\lambda x . x x)$ can be defined as follows (writing 0 instead of zero):

$$
\begin{aligned}
& \Omega: \operatorname{Tm} 0 \\
& \Omega=\operatorname{lam}(\operatorname{var} 0 \cdot \operatorname{var} 0) \cdot \operatorname{lam}(\operatorname{var} 0 \cdot \operatorname{var} 0)
\end{aligned}
$$

It is easy to show that this term does not terminate:

\footnotetext{
${ }^{5}$ In the accompanying code $\llbracket-\rrbracket$ is defined using a data type containing the constructors return, _»=- fail and later, thus ensuring guardedness. These constructors are interpreted in the usual way in a second pass over the result. This technique is explained in detail by Danielsson (2010).
} 
$\Omega$-loops : $\llbracket \Omega \rrbracket[] \approx$ never

$\Omega$-loops $=$ later $(\sharp \Omega$-loops $)$

\section{Type Soundness}

To illustrate how the semantics can be used, let us define a type system and prove type soundness.

I follow Leroy and Grall (2009) and define recursive, simple types coinductively as follows:

$$
\begin{aligned}
\text { data } T y & : \text { Set } \text { where } \\
\text { nat } & : T y \\
\rightarrow \rightarrow- & : \infty T y \rightarrow \infty T y \rightarrow T y
\end{aligned}
$$

Contexts can be defined as vectors of types:

Ctxt $: \mathbb{N} \rightarrow$ Set
Ctxt $n=\operatorname{Vec} T y n$

The type system can then be defined inductively. $\Gamma \vdash t \in \sigma$ means that $t$ has type $\sigma$ in context $\Gamma$ :

$$
\begin{aligned}
& \text { data } \vdash_{-} \epsilon_{-}(\Gamma: \operatorname{Ctxt} n): T m n \rightarrow T y \rightarrow \text { Set where } \\
& \text { con : } \Gamma \vdash \operatorname{con} i \in \text { nat } \\
& \text { var : } \Gamma \vdash \operatorname{var} x \in \text { lookup } x \Gamma \\
& \text { lam : }{ }^{b} \sigma:: \Gamma \vdash t \in{ }^{b} \tau \rightarrow \Gamma \vdash \operatorname{lam} t \in \sigma \rightarrow \tau \\
& { }_{-} \cdot{ }_{-}: \Gamma \vdash t_{1} \in \sigma \rightarrow \tau \rightarrow \Gamma \vdash t_{2} \in{ }^{b} \sigma \rightarrow \\
& \Gamma \vdash t_{1} \cdot t_{2} \in{ }^{b} \tau
\end{aligned}
$$

The use of negative recursive types implies that there are welltyped terms which do not terminate. For instance, $\Omega$ is typeable with any type:

$$
\begin{aligned}
& \Omega \text {-well-typed }:(\tau: T y) \rightarrow[] \vdash \Omega \in \tau \\
& \Omega \text {-well-typed } \tau=-_{-}\{\sigma=\sharp \sigma\}\{\tau=\sharp \tau\} \\
& \text { (lam (var · var)) (lam (var · var)) } \\
& \text { where } \sigma=\sharp \sigma \rightarrow \sharp \tau
\end{aligned}
$$

(Some implicit arguments which Agda could not infer have been given explicitly using the $\{x=\ldots\}$ notation.)

Let us now prove that well-typed programs (closed terms) do not go wrong. It is easy to state what should be proved:

$$
\text { type-soundness : [] } \vdash t \in \sigma \rightarrow \neg(\llbracket t \rrbracket[] \approx \text { fail })
$$

Here $\neg_{-}$is negation $(\neg A=A \rightarrow$ Empty, where Empty is the empty type). As noted by Leroy and Grall it is harder to state type soundness for usual big-step semantics, because such semantics do not distinguish between terms which go wrong and terms which fail to terminate.

We can start by defining a reusable predicate transformer which lifts predicates on $A$ to predicates on $(\text { Maybe } A)_{\perp}$. If Lift $P x$ holds, then we know both that the computation $x$ does not crash, and that if $x$ terminates with a value, then the value satisfies $P$. Lift is defined coinductively as follows:

$$
\begin{aligned}
\text { data Lift }(P & : A \rightarrow \text { Set }):(\text { Maybe } A)_{\perp} \rightarrow \text { Set } \text { where } \\
\text { now-just } & : P x \quad \rightarrow \text { Lift } P(\text { return } x) \\
\text { later } & : \infty\left(\text { Lift } P\left({ }^{b} x\right)\right) \rightarrow \text { Lift } P(\text { later } x)
\end{aligned}
$$

The proof below uses the fact that bind "preserves" Lift:

$$
\begin{aligned}
\text { ¿ऍ-cong_: } & \text { Lift } P x \rightarrow(\{x: A\} \rightarrow P x \rightarrow \text { Lift } Q(f x)) \rightarrow \\
& \text { Lift } Q(x \gg=f)
\end{aligned}
$$

Let us now define some typing predicates for values and computations, introduced mainly as part of the proof of type soundness. $W F_{\mathrm{V}} \sigma v$ means that the value $v$ is well-formed with respect to the type $\sigma$. This relation is defined inductively, mutually with a corresponding relation for environments:

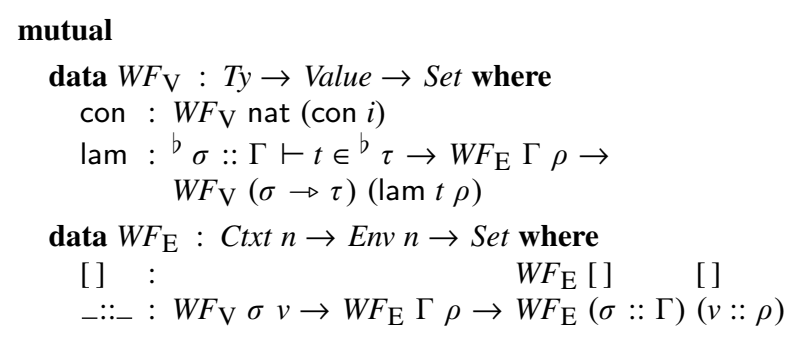

The most interesting case above is that for closures. A closure lam $t \rho$ is well-formed with respect to $\sigma \rightarrow \tau$ if there is a context $\Gamma$ such that $\Gamma \vdash \operatorname{lam} t \in \sigma \rightarrow \tau$ and $\rho$ is well-formed with respect to $\Gamma$. The predicates are related by the following unsurprising lemma:

$$
\begin{aligned}
\text { lookup }_{\mathrm{wf}}: & (x: \text { Fin } n) \rightarrow W_{\mathrm{E}} \Gamma \rho \rightarrow \\
& \text { WF } F_{\mathrm{V}}(\text { lookup } x \Gamma)(\text { lookup } x \rho)
\end{aligned}
$$

We can use the predicate transformer introduced above to lift $W F_{\mathrm{V}}$ to computations:

$$
\begin{aligned}
& W F_{\perp}: T y \rightarrow(\text { Maybe Value })_{\perp} \rightarrow \text { Set } \\
& W F_{\perp} \sigma x=\text { Lift }\left(W F_{\mathrm{V}} \sigma\right) x
\end{aligned}
$$

Non-terminating computations are well-formed, and terminating computations are well-formed if they are successful (not nothing) and the value is well-formed. The following lemma implies that type soundness can be established by showing that $\llbracket t \rrbracket[]$ is wellformed:

$$
\begin{aligned}
& \text { does-not-go-wrong : } W F_{\perp} \sigma x \rightarrow \neg(x \approx \text { fail) } \\
& \text { does-not-go-wrong (now-just } \left.\_\right)() \\
& \text {does-not-go-wrong (later } w f) \quad\left(\operatorname{later}^{1} e q\right)= \\
& \quad \text { does-not-go-wrong }\left({ }^{b} w f\right) e q
\end{aligned}
$$

Recall that negation is a function into the empty type. The lemma is proved by structural recursion: induction on the structure of the proof of $x \approx$ fail. The first clause contains an "absurd pattern", (), to indicate that there is no constructor application of type return $v \approx$ fail.

We can now prove the main lemma, which states that the computations resulting from evaluating well-typed terms in wellformed environments are well-formed. This lemma uses the same form of nested corecursion/structural recursion as the definition of the semantics:

$$
\begin{aligned}
& \text { mutual } \\
& \llbracket \rrbracket_{\mathrm{wf}}: \Gamma \vdash t \in \sigma \rightarrow W F_{\mathrm{E}} \Gamma \rho \rightarrow W F_{\perp} \sigma(\llbracket t \rrbracket \rho) \\
& \llbracket \rrbracket_{\mathrm{wf}} \text { con } \quad \rho_{\mathrm{wf}}=\text { now-just con } \\
& \llbracket \rrbracket_{\mathrm{wf}}(\operatorname{var}\{x=x\}) \rho_{\mathrm{wf}}=\text { now-just }\left(\text { lookup }_{\mathrm{wf}} x \rho_{\mathrm{wf}}\right) \\
& \llbracket \rrbracket_{\mathrm{Wf}}\left(\operatorname{lam} t_{\epsilon}\right) \quad \rho_{\mathrm{wf}}=\text { now-just }\left(\operatorname{lam} t_{\in} \rho_{\mathrm{wf}}\right) \\
& \llbracket \rrbracket_{\mathrm{wf}}\left(t_{1 \in} \cdot t_{2 \in}\right) \quad \rho_{\mathrm{wf}}= \\
& \llbracket \rrbracket_{\mathrm{wf}} t_{1 \in} \rho_{\mathrm{wf}} \gg \text {-cong } \lambda f_{\mathrm{wf}} \rightarrow \\
& \llbracket \rrbracket_{\mathrm{wf}} t_{2 \in} \rho_{\mathrm{wf}} \gg \text {-cong } \lambda v_{\mathrm{wf}} \rightarrow \\
& \bullet_{\mathrm{wf}} f_{\mathrm{wf}} v_{\mathrm{wf}} \\
& \bullet_{\mathrm{wf}}: W F_{\mathrm{V}}(\sigma \rightarrow \tau) f \rightarrow W F_{\mathrm{V}}\left({ }^{b} \sigma\right) v \rightarrow \\
& W F_{\perp}\left({ }^{b} \tau\right)(f \bullet v)
\end{aligned}
$$

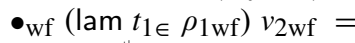

$$
\begin{aligned}
& \text { later }\left(\sharp \llbracket \rrbracket_{\mathrm{wf}} t_{1 \in}\left(v_{2 \mathrm{wf}}:: \rho_{1 \mathrm{wf}}\right)\right)
\end{aligned}
$$

The implicit variable pattern $\{x=x\}$ is used to bind the variable $x$, which is used on the right-hand side.

Finally we can conclude:

$$
\begin{aligned}
& \text { type-soundness }:[] \vdash t \in \sigma \rightarrow \neg(\llbracket t \rrbracket[] \approx \text { fail }) \\
& \text { type-soundness } t_{\in}=\text { does-not-go-wrong }\left(\mathbb{\llbracket} \rrbracket_{\mathrm{wf}} t_{\in}[]\right)
\end{aligned}
$$

Note that there is only one case for application in the proof above (plus one sub-case in $\bullet$ wf). 
The proof of type soundness is formulated for a functional semantics defined using environments and closures, whereas Leroy and Grall (2009) prove type soundness for relational semantics defined using substitutions. I have chosen to use environments and closures in this paper to avoid distracting details related to substitutions. However, given an implementation of the operation which substitutes a term for variable zero it is easy to define a substitutionbased functional semantics using the partiality monad, and given a proof showing that this operation preserves types it is easy to adapt the proof above to this semantics. See the accompanying code for details.

The proof above can be compared to a typical type soundness proof formulated for a relational, substitution-based smallstep semantics. Such a proof often amounts to proving progress and preservation:

$$
\begin{aligned}
& \text { progress : [] } \vdash t \in \sigma \rightarrow \text { Value } t \uplus \exists \lambda t^{\prime} \rightarrow t \leadsto t^{\prime} \\
& \text { preservation : [ [ } \vdash t \in \sigma \rightarrow t \leadsto t^{\prime} \rightarrow[] \vdash t^{\prime} \in \sigma
\end{aligned}
$$

Here Value $t$ means that $t$ is a value, _ $\sim_{-}$is the small-step relation, and $\exists \lambda t^{\prime} \rightarrow \ldots$ can be read as "there exists a $t^{\prime}$ such that...". Given these two lemmas one can prove type soundness using classical reasoning (Leroy and Grall 2009):

$$
\begin{aligned}
\text { type-soundness : [ ] } \vdash t \in \sigma \rightarrow & \\
t & \sim \infty \uplus \exists \lambda t^{\prime} \rightarrow t \sim^{\star} t^{\prime} \times \text { Value } t^{\prime}
\end{aligned}
$$

Here $\_\sim^{\star}-$ is the reflexive transitive closure of $\_\sim_{-}, t \leadsto \infty$ means that $t$ can reduce forever, and _- $\times_{-}$can be read as "and". (Note that this statement of type soundness is inappropriate for non-deterministic languages, as it does not rule out the possibility of crashes.) The lemma $\llbracket \rrbracket_{w f}$ above can be seen as encompassing both progress and preservation, plus the combination of these two lemmas into type soundness. This combination does not need to involve classical reasoning, because $W F_{\perp}$ is defined coinductively.

\section{The Semantics are Classically Equivalent}

Let us now prove that the semantics given in Section 3 is classically equivalent to a relational semantics.

The semantics given in Figures 1-2 can be adapted to a setting with well-scoped terms and de Bruijn indices in the following way:

$$
\begin{aligned}
& \text { data } \_\vdash_{-} \Downarrow_{-}(\rho: \text { Env } n): \operatorname{Tm} n \rightarrow \text { Value } \rightarrow \text { Set where } \\
& \text { con : } \rho \vdash \operatorname{con} i \Downarrow \operatorname{con} i \\
& \operatorname{var}: \rho \vdash \operatorname{var} x \Downarrow \text { lookup } x \rho \\
& \operatorname{lam}: \rho \vdash \operatorname{lam} t \Downarrow \operatorname{lam} t \rho \\
& \text { app : } \rho \vdash t_{1} \Downarrow \operatorname{lam} t^{\prime} \rho^{\prime} \rightarrow \rho \vdash t_{2} \Downarrow v^{\prime} \rightarrow \\
& v^{\prime}:: \rho^{\prime} \vdash t^{\prime} \Downarrow v \rightarrow \rho \vdash t_{1} \cdot t_{2} \Downarrow v \\
& \text { data_} \vdash \_(\rho: E n v n): T m n \rightarrow \text { Set where } \\
& \operatorname{app}^{1}: \infty\left(\rho \vdash t_{1} \Uparrow\right) \rightarrow \rho \vdash t_{1} \cdot t_{2} \Uparrow \\
& \operatorname{app}^{\mathrm{r}}: \rho \vdash t_{1} \Downarrow v \rightarrow \infty\left(\rho \vdash t_{2} \Uparrow\right) \rightarrow \rho \vdash t_{1} \cdot t_{2} \Uparrow \\
& \text { app }: \rho \vdash t_{1} \Downarrow \operatorname{lam} t^{\prime} \rho^{\prime} \rightarrow \rho \vdash t_{2} \Downarrow v^{\prime} \rightarrow \\
& \infty\left(v^{\prime}:: \rho^{\prime} \vdash t^{\prime} \Uparrow\right) \rightarrow \rho \vdash t_{1} \cdot t_{2} \Uparrow \\
& \vdash_{-} \downarrow: \text { Env } n \rightarrow \operatorname{Tm} n \rightarrow \text { Set } \\
& \rho \vdash t \dot{z}=\neg(\exists \lambda v \rightarrow \rho \vdash t \Downarrow v) \times \neg(\rho \vdash t \Uparrow)
\end{aligned}
$$

Note that $\_\vdash_{-} \Downarrow_{-}$is defined inductively and $\_\vdash_{-} \Uparrow$ coinductively.

How should we state the equivalence of $\_\vdash_{-} \downarrow \ell_{-} \vdash_{-} \Uparrow / \vdash_{-} \vdash_{-}$ and $\llbracket-\rrbracket$ ? The following may seem like a suitable statement:

$$
\begin{aligned}
& \rho \vdash t \Downarrow v \quad \Leftrightarrow \quad \llbracket t \rrbracket \rho \approx \text { return } v \\
& \rho \vdash t \Uparrow \quad \Leftrightarrow \quad \llbracket t \rrbracket \rho \approx \text { never } \\
& \rho \vdash t \downarrow
\end{aligned}
$$

However, in a constructive setting one cannot prove that $\llbracket t \rrbracket \rho \approx$ never implies $\rho \vdash t \Uparrow$. To see why, let us try. Assume that we have a proof $p$ of type $\llbracket t_{1} \cdot t_{2} \rrbracket \rho \approx$ never. Now we need to construct a proof starting with either app $p^{1}, a p p^{r}$ or app. In order to do this we need to know whether $t_{1}$ terminates or not, but this is not decidable given only the proof $p$. It also seems unlikely that we can prove that $\rho \vdash t$ implies $\llbracket t \rrbracket \rho \approx$ fail: one might imagine that this can be proved by just executing $\llbracket t \rrbracket \rho$ until it terminates and then performing a case analysis, but the fact that $t$ does not fail to terminate is not (obviously) enough to convince Agda that it does terminate.

We can avoid these issues by assuming the following form of excluded middle, which states that everything (in Set) is decidable:

$$
\begin{aligned}
& E M: \operatorname{Set}_{1} \\
& E M=(A: \text { Set }) \rightarrow A \uplus \neg A
\end{aligned}
$$

We end up with the following six proof obligations:

$$
\begin{aligned}
& \rho \vdash t \Downarrow v \quad \rightarrow \llbracket t \rrbracket \rho \approx \text { return } v \\
& \rho \vdash t \Uparrow \rightarrow \llbracket t \rrbracket \rho \approx \text { never } \\
& \llbracket t \rrbracket \rho \approx \text { return } v \rightarrow \rho \vdash t \Downarrow v \\
& E M \rightarrow \llbracket t \rrbracket \rho \approx \text { never } \rightarrow \rho \vdash t \Uparrow \\
& E M \rightarrow \rho \vdash t \downarrow \rightarrow \llbracket t \rrbracket \rho \approx \text { fail }
\end{aligned}
$$

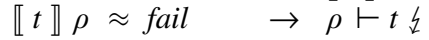

The last two follow easily from the previous ones, so let us focus on the first four:

1. Given $p: \rho \vdash t \Downarrow v$ it is easy to prove $\llbracket t \rrbracket \rho \approx$ return $v$ by recursion on the structure of $p$.

The only interesting case is application. Let us introduce the following abbreviation:

$$
x_{1} \llbracket \cdot \rrbracket x_{2}=x_{1} \gg \lambda v_{1} \rightarrow x_{2} \gg \lambda v_{2} \rightarrow v_{1} \bullet v_{2}
$$

We can then proceed as follows (using the same names as in the app constructor's type signature):

$$
\begin{aligned}
& \begin{array}{ll}
\llbracket t_{1} \cdot t_{2} \rrbracket \rho & \\
\llbracket t_{1} \rrbracket \rho & \llbracket \cdot \rrbracket \llbracket t_{2} \rrbracket \rho \approx
\end{array} \\
& \text { return }\left(\operatorname{lam} t^{\prime} \rho^{\prime}\right) \llbracket \cdot \rrbracket \text { return } v^{\prime} \gtrsim \\
& \llbracket t^{\prime} \rrbracket\left(v^{\prime}:: \rho^{\prime}\right) \\
& \text { return } v
\end{aligned}
$$

The inductive hypothesis is used twice in the second step and once in the last one.

2. One can prove that $\rho \vdash t \Uparrow$ implies $\llbracket t \rrbracket \rho \approx$ never using corecursion plus an inner recursion on the structure of $t$.

In the case of the app constructor we can proceed as follows:

$$
\begin{aligned}
& \llbracket t_{1} \cdot t_{2} \rrbracket \rho \\
& \llbracket t_{1} \rrbracket \rho \quad \llbracket \cdot \rrbracket \llbracket t_{2} \rrbracket \rho, \gtrsim \\
& \text { return }\left(\operatorname{lam} t^{\prime} \rho^{\prime}\right) \llbracket \cdot \rrbracket \text { return } v^{\prime} \cong \\
& \text { later }\left(\sharp \llbracket t^{\prime} \rrbracket\left(v^{\prime}:: \rho^{\prime}\right)\right) \\
& \text { never }
\end{aligned}
$$

The second step uses (1) twice, once for $p_{1}: \rho \vdash t_{1} \Downarrow$ lam $t^{\prime} \rho^{\prime}$ and once for $p_{2}: \rho \vdash t_{2} \Downarrow v^{\prime}$, plus the fact that $x \approx$ now $v$ implies that $x \gtrsim$ now $v$. The last step uses the coinductive hypothesis (under a guard) for $p_{3}: v^{\prime}:: \rho^{\prime} \vdash t^{\prime} \Uparrow$.

The app ${ }^{1}$ case is different:

$$
\begin{aligned}
& \llbracket t_{1} \cdot t_{2} \rrbracket \rho \\
& \llbracket t_{1} \rrbracket \rho \llbracket \cdot \rrbracket \llbracket t_{2} \rrbracket \rho \approx \\
& \text { never } \llbracket \cdot \rrbracket \llbracket t_{2} \rrbracket \rho \cong \\
& \text { never }
\end{aligned}
$$

The last step uses the fact that never is a left zero of bind. The second step uses the inductive hypothesis for $p: \rho \vdash t_{1} \Uparrow$; note that $t_{1}$ is structurally smaller than $t_{1} \cdot t_{2}$, and that this call is not guarded. 
The app ${ }^{\mathrm{r}}$ case is similar to the app ${ }^{1}$ one, and omitted.

Note that the use of transitivity in this proof is safe, as discussed in Section 2.

3. Given $p: \llbracket t \rrbracket \rho \approx$ return $v$ one can observe that $p$ cannot contain the constructors later or later ${ }^{\mathrm{r}}$ : it must have the form later $^{1}\left(\ldots\right.$ (later ${ }^{1}$ now) ...), with a finite number of later ${ }^{1}$ constructors - one for every $\beta$-reduction in the computation of $\llbracket t \rrbracket \rho$. Let the size of $p$ be this number. One can prove that $\llbracket t \rrbracket \rho \approx$ return $v$ implies $\rho \vdash t \Downarrow v$ by complete induction on this size.

Only the application case is interesting. We can prove the following inversion lemma:

$$
\begin{aligned}
& (x \gg f) \approx \text { return } v \rightarrow \\
& \quad \exists \lambda v^{\prime} \rightarrow\left(x \approx \text { return } v^{\prime}\right) \times\left(f v^{\prime} \approx \text { return } v\right)
\end{aligned}
$$

Here the size of the left-hand proof is equal to the sum of the sizes of the two right-hand proofs. If we have $\llbracket t_{1} \cdot t_{2} \rrbracket \rho \approx$ return $v$, then we can use inversion twice plus case analysis to deduce that $\llbracket t_{1} \rrbracket \rho \approx \operatorname{return}\left(\operatorname{lam} t^{\prime} \rho^{\prime}\right)$ and $\llbracket t_{2} \rrbracket \rho \approx$ return $v^{\prime}$ for some $t^{\prime}, \rho^{\prime}, v^{\prime}$ such that $\llbracket t^{\prime} \rrbracket\left(v^{\prime}:: \rho^{\prime}\right) \approx$ return $v$. We can finish by applying app to three instances of the inductive hypothesis, after making sure that the proofs are small enough.

This proof is a bit awkward when written out in detail, due to the use of sizes.

4. Finally we should prove that excluded middle and $\llbracket t \rrbracket \rho \approx$ never imply $\rho \vdash t \Uparrow$. This can be proved using corecursion.

As before the only interesting case is application. We can prove the following inversion lemma by using excluded middle:

$$
\begin{aligned}
& (x \gg f) \approx \text { never } \rightarrow \\
& x \approx \text { never } \uplus \\
& \quad \exists \lambda v \rightarrow(x \approx \text { return } v) \times(f v \approx \text { never })
\end{aligned}
$$

If $x \gg f$ does not terminate, then either $x$ fails to terminate, or $x$ terminates with a value $v$ and $f v$ does not terminate. Given a proof of $\llbracket t_{1} \cdot t_{2} \rrbracket \rho \approx$ never we can use inversion twice to determine which of app $\mathrm{app}^{\mathrm{r}}$ and app to emit, in each case continuing corecursively (and in the latter two cases also using (3)).

\section{Virtual Machine}

This section defines a virtual machine (VM), following Leroy and Grall (2009) but defining the semantics functionally instead of relationally, and using a well-scoped approach. (The accompanying code contains a relational semantics and a proof showing that it is equivalent to the functional one.)

The VM is stack-based, and uses the following instructions:

\section{mutual}

$$
\begin{aligned}
& \text { data } \operatorname{Instr}(n: \mathbb{N}): \text { Set where } \\
& \text { var : Fin } n \rightarrow \text { Instr } n \quad-\text { Push variable. } \\
& \text { con : } \mathbb{N} \rightarrow \text { Instr } n \quad-\text { Push constant. } \\
& \text { clo : Code }(1+n) \rightarrow \text { Instr } n \quad-\text { Push closure. } \\
& \text { app : } \quad \operatorname{Instr} n \text {-- Apply function. } \\
& \text { ret : Instr } n \text {-- Return. } \\
& \text { Code }: \mathbb{N} \rightarrow \text { Set } \\
& \text { Code } n=\text { List }(\text { Instr } n)
\end{aligned}
$$

Instructions of type Instr $n$ have at most $n$ free variables. The type family Code consists of sequences of instructions.

Values and environments (VM-Value and VM-Env) are defined as in Section 3, but using Code instead of Tm in the definition of closures. Stacks contain values and return frames:

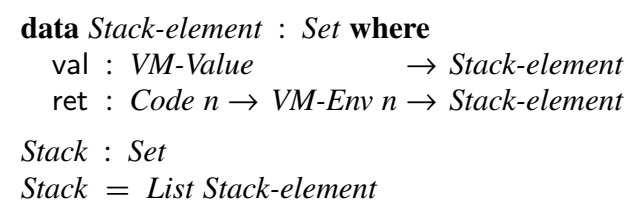

The VM operates on states containing three components, the code, a stack, and an environment:

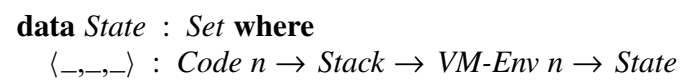

The result of running the VM one step, starting in a given state, is either a new state, normal termination with a value, or abnormal termination (a crash):

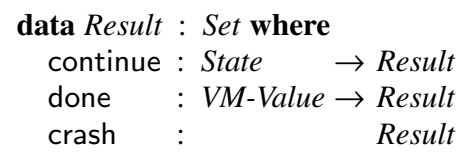

The function step (see Figure 3) shows how the result is computed. Given step it is easy to define the VM's semantics corecursively:

$$
\begin{aligned}
& \text { exec }: \text { State } \rightarrow(\text { Maybe VM-Value })_{\perp} \\
& \text { exec } s \text { with step } s \\
& \text {... continue } s^{\prime}=\operatorname{later}\left(\sharp \text { exec } s^{\prime}\right) \\
& \text {... done } v=\text { return } v \\
& \text {.. } \mid \text { crash }=\text { fail }
\end{aligned}
$$

In a state $s$, run step $s$. If the result is continue $s^{\prime}$, continue running from $s^{\prime}$; if it is done $v$, return $v$; and if it is crash, fail.

The function exec is an example of a functional, small-step operational semantics. As before it is clear that the semantics is deterministic and computable, and just as with a relational smallstep semantics we avoid duplication of rules. However, the use of a wild-card in the last clause of step means that it is possible to forget a rule. If we tried to omit one of the clauses from the definition of $\llbracket-\rrbracket$ (Section 3), then the definition would be rejected, but this is not the case for the first six clauses of step.

\section{Compiler Correctness}

Let us now define a compiler from $\mathrm{Tm}$ to Code and prove that it preserves the semantics of the input program. The definition follows Leroy and Grall (2009), but uses a code continuation to avoid the use of list append and some proof overhead (Hutton 2007, Section 13.7):

$$
\begin{aligned}
& \operatorname{comp}: \text { Tm } n \rightarrow \text { Code } n \rightarrow \text { Code } n \\
& \operatorname{comp}(\operatorname{con} i) c=\operatorname{con} i:: c \\
& \operatorname{comp}(\operatorname{var} x) c=\operatorname{var} x:: c \\
& \operatorname{comp}(\operatorname{lam} t) c=\operatorname{clo}(\operatorname{comp} t[\text { ret }]):: c \\
& \operatorname{comp}\left(t_{1} \cdot t_{2}\right) c=\operatorname{comp} t_{1}\left(\operatorname{comp} t_{2}(\operatorname{app}:: c)\right)
\end{aligned}
$$

We can also "compile" values:

$$
\begin{aligned}
& \operatorname{comp}_{\mathrm{v}}: \text { Value } \rightarrow \text { VM-Value } \\
& \operatorname{comp}_{\mathrm{v}}(\operatorname{con} i)=\operatorname{con} i \\
& \operatorname{comp}_{\mathrm{v}}(\operatorname{lam} t \rho)=\operatorname{lam}\left(\operatorname{comp} t[\text { ret] })\left(\operatorname{map} \operatorname{comp}_{\mathrm{v}} \rho\right)\right.
\end{aligned}
$$

I state compiler correctness as follows:

$$
\begin{aligned}
\text { correct }: & (t: \operatorname{Tm} 0) \rightarrow \\
& \text { exec }\langle\text { comp } t[],[],[]\rangle \approx \\
& \left(\llbracket t \rrbracket[] \gg \lambda v \rightarrow \operatorname{return}\left(\operatorname{comp}_{\mathrm{v}} v\right)\right)
\end{aligned}
$$

Given a closed term $t$, the result of running the corresponding compiled code (comp $t$ []) on the VM (starting with an empty stack and environment), should be the same as evaluating the term (in 


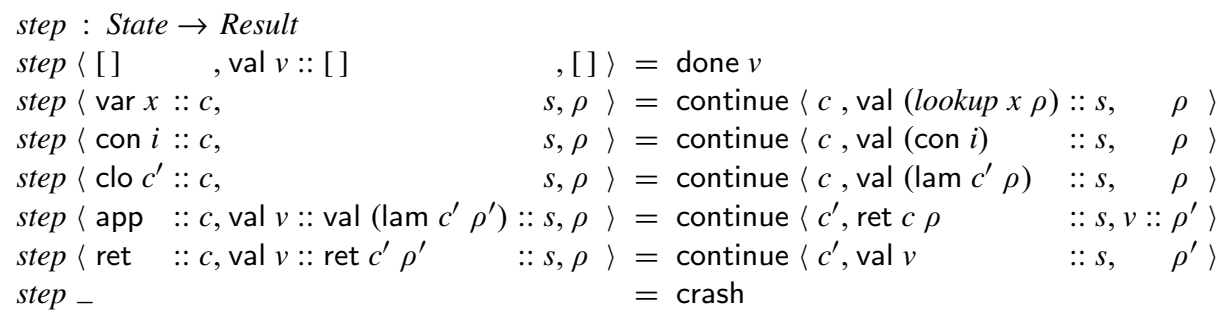

Figure 3. A function which computes the result of running the virtual machine one step from a given state.

an empty environment) and, if evaluation terminates with a value, return the "compiled" variant of this value.

We can compare this statement to a corresponding statement phrased for relational semantics:

$$
\begin{aligned}
& \left([] \vdash t \Downarrow v \Leftrightarrow\langle\operatorname{comp} t[],[],[]\rangle \sim^{\star}\right. \\
& \left.\left\langle[], \text { val }\left(\operatorname{comp}_{\mathrm{v}} v\right)::[],[]\right\rangle\right) \times \\
& ([] \vdash t \Uparrow \Leftrightarrow\langle\operatorname{comp} t[],[],[]\rangle \sim \infty) \quad \times \\
& ([] \vdash t \xi \Leftrightarrow\langle\operatorname{comp} t[],[],[]\rangle \sim \hat{k})
\end{aligned}
$$

Here $\_{ }_{-}:$State $\rightarrow$ State $\rightarrow$ Set is the VM's small-step relation, $\sim^{\star}{ }^{\star}$ its reflexive transitive closure, $s \leadsto \infty$ means that there is an infinite transition sequence starting in $s$, and $s \leadsto \dot{k}$ means that there is a "stuck" transition sequence starting in $s$ (i.e., a sequence which cannot be extended further, and which does not end with a state of the form ( [], val _ :: [], [] 〉). I prefer the statement of correct above: I find it easier to understand and get correct.

Let us now prove correct. In order to do this the statement can be generalised as follows:

$$
\begin{aligned}
& \text { correct' }: \\
& \quad(t: \text { Tm } n)\left\{k: \text { Value } \rightarrow(\text { Maybe VM-Value })_{\perp}\right\} \\
& \quad(\text { hyp }:(v: \text { Value }) \rightarrow \\
& \left.\quad \text { exec }\left\langle c, \text { val }\left(\operatorname{comp}_{\mathrm{v}} v\right):: s, \text { map } \operatorname{comp}_{\mathrm{v}} \rho\right\rangle \approx k v\right) \rightarrow \\
& \text { exec }\left\langle\operatorname{comp} t c, s, \text { map comp } p_{\mathrm{v}} \rho\right\rangle \approx(\llbracket t \rrbracket \rho \gg k)
\end{aligned}
$$

This statement is written in continuation-passing style to avoid some uses of transitivity (which can be problematic, as discussed in Section 2). The statement is proved mutually with the following one:

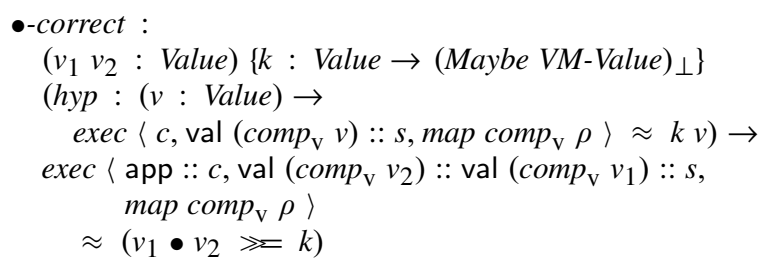

The statements can be proved using the same recursion structure as $\llbracket-\rrbracket_{\text {CPS }} / \bullet_{\text {CPS }}-$ mixed corecursion/structural recursion.

The interesting case of correct ${ }^{\prime}$ is application, where we can proceed as follows (with safe uses of transitivity):

$$
\begin{aligned}
& \text { exec }\left\langle\operatorname { c o m p } _ { 1 } \left(\operatorname{comp}_{2}\left(\text { app :: c)), s, map } \operatorname{comp}_{\mathrm{v}} \rho\right\rangle \quad \approx\right.\right. \\
& \llbracket t_{1} \rrbracket \rho \gg \lambda v_{1} \rightarrow \llbracket t_{2} \rrbracket \rho \gg \lambda v_{2} \rightarrow v_{1} \bullet v_{2} \gg k \cong \\
& \llbracket t_{1} \rrbracket \rho \gg \lambda v_{1} \rightarrow\left(\llbracket t_{2} \rrbracket \rho \gg \lambda v_{2} \rightarrow v_{1} \bullet v_{2}\right) \gg k \cong \\
& \left(\llbracket t_{1} \rrbracket \rho \gg \lambda v_{1} \rightarrow \llbracket t_{2} \rrbracket \rho \gg \lambda v_{2} \rightarrow v_{1} \bullet v_{2}\right) \gg k \cong \\
& \llbracket t_{1} \cdot t_{2} \rrbracket \rho \gg k
\end{aligned}
$$

The last three steps use associativity of bind twice. (These uses of associativity could have been avoided by using continuationpassing style instead of bind when defining the semantics. See the accompanying code.) The first step is more complicated. Here is its proof term: correct' $t_{1}\left(\lambda v_{1} \rightarrow\right.$ correct $^{\prime} t_{2}\left(\lambda v_{2} \rightarrow \bullet\right.$-correct $v_{1} v_{2}$ hyp $\left.)\right)$

First an appeal to the inductive hypothesis $\left(t_{1}\right.$ is structurally smaller than $\left.t_{1} \cdot t_{2}\right)$, then, in the continuation, another appeal to the inductive hypothesis, and finally, in the nested continuation, a use of $\bullet$-correct.

The interesting case of $\bullet$-correct is when $v_{1}$ is a closure, lam $t_{1} \rho_{1}$, in which case we need to prove that

$$
\begin{aligned}
& \text { exec }\left\langle\text { app }:: c \text {, val }\left(\operatorname{comp}_{\mathrm{v}} v_{2}\right):: \text { val }\left(\operatorname{comp}_{\mathrm{v}}\left(\operatorname{lam} t_{1} \rho_{1}\right)\right):: s\right. \text {, } \\
& \left.\operatorname{map~comp~}_{\mathrm{v}} \rho\right\rangle
\end{aligned}
$$

is weakly bisimilar to

$$
\operatorname{lam} t_{1} \rho_{1} \bullet v_{2} \gg=k \text {. }
$$

We can start by emitting a later constructor and suspension:

$$
\text { later }(\sharp \text { ?) }
$$

The question mark should be replaced by a proof showing that

$$
\begin{gathered}
\text { exec }\left\langle\operatorname { c o m p } _ { 1 } \left[\text { ret], ret } c\left(\operatorname{map} \operatorname{comp}_{\mathrm{v}} \rho\right):: s,\right.\right. \\
\left.\operatorname{map}_{\operatorname{comp}}\left(v_{2}:: \rho_{1}\right)\right\rangle
\end{gathered}
$$

is weakly bisimilar to

$$
\llbracket t_{1} \rrbracket\left(v_{2}:: \rho_{1}\right) \gg k \text {. }
$$

This can be proved by appeal to the coinductive hypothesis:

$$
\text { correct' }^{\prime} t_{1}\left(\lambda v \rightarrow \text { later }^{1}(\text { hyp } v)\right)
$$

Here the use of later ${ }^{1}$ corresponds to the reduction of

$$
\begin{gathered}
\text { exec }\left\langle[\text { ret }], \text { val }\left(\operatorname{comp}_{\mathrm{v}} v\right):: \text { ret } c\left(\operatorname{map}_{\operatorname{comp}} \rho\right):: s,\right. \\
\left.\operatorname{map} \operatorname{comp}_{\mathrm{v}}\left(v_{2}:: \rho_{1}\right)\right\rangle
\end{gathered}
$$

to

exec $\left\langle c\right.$, val $\left.\left(\operatorname{comp}_{\mathrm{v}} v\right):: s, \operatorname{map}_{\operatorname{comp}} \rho\right\rangle$,

which has the right form for the use of hyp.

The proof sketch above-and especially the compact proof terms - may look a bit bewildering. Fortunately one does not have to understand every detail of a machine-checked proof. It is more important to understand the statement of the theorem. ${ }^{6}$ Furthermore, the writer of the proof has the support of a proof assistant, that in my case provided much help with the construction of the proof terms.

The proof above can be compared to that of Leroy and Grall (2009), who prove the following two implications (in their slightly different setting):

$$
\begin{gathered}
{[] \vdash t \Downarrow v \rightarrow\langle\operatorname{comp} t[],[],[]\rangle \sim^{\star}} \\
\left\langle[], \operatorname{val}\left(\operatorname{comp}_{\mathrm{v}} v\right)::[],[]\right\rangle \\
{[] \vdash t \Uparrow \rightarrow\langle\operatorname{comp} t[],[],[]\rangle \sim \infty}
\end{gathered}
$$

\footnotetext{
${ }^{6}$ With the caveat that one should not put too much trust into Agda, which is a very experimental system.
} 
Consider application. In the proof above there is one case for application, with two sub-cases, one for crashes and one for closures. In the proof of the two implications there are four cases for application: one in case of termination and three for non-terminating applications. The rule duplication in the semantics shows up as rule duplication in the proof.

\section{Non-determinism}

The compiler correctness statement used above is sometimes too restrictive (Leroy 2009). For instance, evaluation order may be left up to the compiler. This section illustrates how this kind of situation can be handled by defining a non-deterministic language, and implementing a compiler that implements one out of many possible semantics for this language.

The syntax of the language defined in Section 3 is extended with a term-former for non-deterministic choice:

$$
\text { -_}_{-}: \operatorname{Tm} n \rightarrow \operatorname{Tm} n \rightarrow \operatorname{Tm} n
$$

The semantic domain is now the maybe monad transformer applied to the partiality monad transformer $(\lambda M A . v X . M(A \uplus X)$ for strictly positive monads $M)$ applied to a non-determinism monad ( $\lambda A . \mu X . A \uplus X \times X$; Moggi (1990)), implemented monolithically as follows:

$$
\begin{aligned}
& \text { data } D(A: \text { Set }) \text { : Set where } \\
& \text { fail : } D A \\
& \text { return : } A \rightarrow D A \\
& \text {-l_ }: D A \rightarrow D A \rightarrow D A \\
& \text { later }: \infty(D A) \rightarrow D A \\
& { }_{-}: D A \rightarrow(A \rightarrow D B) \rightarrow D B \\
& \text { fail } \gg f=\text { fail } \\
& \text { return } x \gg f=f x \\
& \left(x_{1} \mid x_{2}\right) \gg f=\left(x_{1} \gg f\right) \mid\left(x_{2} \gg f\right) \\
& \text { later } x \gg=f=\text { later }(\sharp(b x \gg=f))
\end{aligned}
$$

As before the monad laws hold up to strong bisimilarity, which can be defined as follows:

$$
\begin{aligned}
& \text { data } \cong_{-}: D A \rightarrow D A \rightarrow \text { Set } \text { where } \\
& \text { fail : fail } \cong \text { fail } \\
& \text { return : } \quad \text { return } x \cong \text { return } x \\
& \text { - }_{-}: x_{1} \cong y_{1} \rightarrow x_{2} \cong y_{2} \rightarrow x_{1}\left|x_{2} \cong y_{1}\right| y_{2} \\
& \text { later : } \infty\left({ }^{b} x \cong{ }^{b} y\right) \quad \rightarrow \text { later } x \cong \text { later } y
\end{aligned}
$$

Finally we can extend the semantics by adding a clause for choice (note that $\left.{ }_{-}\right|_{-}$is overloaded):

$$
\llbracket t_{1}\left|t_{2} \rrbracket \rho=\llbracket t_{1} \rrbracket \rho\right| \llbracket t_{2} \rrbracket \rho
$$

It may be worth pointing out that now the semantics is no longer deterministic, despite being defined as a function.

As an example we can define a call-by-value fixpoint combinator $(Z=\lambda f .(\lambda g . f(\lambda x . g g x))(\lambda g . f(\lambda x . g g x)))$ and a nondeterministic non-terminating term $(t=Z(\lambda f x . f x \mid f x) 0)$ :

$$
\begin{aligned}
& Z: \operatorname{Tm} 0 \\
& Z=\operatorname{lam}(h \cdot h) \\
& \quad \text { where } h=\operatorname{lam}(\operatorname{var} 1 \cdot \operatorname{lam}(\operatorname{var} 1 \cdot \operatorname{var} 1 \cdot \operatorname{var} 0)) \\
& t: \operatorname{Tm} 0 \\
& t=Z \cdot \operatorname{lam}(\operatorname{lam}(\operatorname{var} 1 \cdot \operatorname{var} 0 \mid \operatorname{var} 1 \cdot \operatorname{var} 0)) \cdot \operatorname{con} 0
\end{aligned}
$$

The semantics of $t, \llbracket t \rrbracket[]$, is strongly bisimilar to $t$-sem:

$$
\text { t-sem : D Value }
$$$$
t \text {-sem }=\text { later }(\sharp \text { later }(\sharp \text { later }(\sharp \text { later }(\sharp(t \text {-sem } \mid t \text {-sem })))))
$$

The virtual machine is unchanged, so the compiler correctness statement will relate deterministic and non-deterministic computa- tions. To do this we can use the following variant of weak bisimilarity:

$$
\begin{aligned}
& \text { data } \approx_{-}^{\in}:(\text { Maybe } A)_{\perp} \rightarrow D A \rightarrow \text { Set } \text { where } \\
& \text { fail : now nothing } \approx^{\in} \text { fail } \\
& \text { return : now (just } x \text { ) } \approx \in \operatorname{return} x \\
& \left.\right|^{1}: x \approx^{\epsilon} y_{1} \rightarrow x \approx^{\epsilon} y_{1} \mid y_{2} \\
& \left.\right|^{\mathrm{r}} \quad: x \approx^{\epsilon} y_{2} \rightarrow x \approx^{\epsilon} y_{1} \mid y_{2} \\
& \text { later }: \infty\left({ }^{b} x \approx{ }^{\dagger} y\right) \rightarrow \text { later } x \approx^{\in} \text { later } y \\
& \text { later }{ }^{1}: \quad b^{b} \approx^{\in} y \rightarrow \text { later } x \approx \in \quad y \\
& \text { later }^{\mathrm{r}}: \quad x \approx \mathrm{f}^{\in} y \rightarrow \quad x \approx \in \text { later } y
\end{aligned}
$$

You can read $x \approx^{\in} y$ as " $x$ implements one of the allowed semantics of $y$ ".

Compiler correctness can now be stated as follows:

$$
\begin{aligned}
\text { correct }: & (t: \operatorname{Tm} 0) \rightarrow \\
& \text { exec }\langle\operatorname{comp} t[],[],[]\rangle \approx \in \\
& \llbracket t \rrbracket[] \gg \lambda v \rightarrow \operatorname{return}\left(\operatorname{comp}_{\mathrm{v}} v\right)
\end{aligned}
$$

If we extend the compiler in the following way, then we can prove that it is correct using an argument which is very similar to that in Section 7:

$$
\operatorname{comp}\left(t_{1} \mid t_{2}\right) c=\operatorname{comp}_{1} c
$$

We can also prove type soundness for the non-deterministic language, using the type system from Section 4 extended with the following rule:

$$
{ }_{-} \mathrm{I}_{-}: \Gamma \vdash t_{1} \in \sigma \rightarrow \Gamma \vdash t_{2} \in \sigma \rightarrow \Gamma \vdash t_{1} \mid t_{2} \in \sigma
$$

Type soundness can be stated using $\_\approx^{\epsilon}$. Type-correct terms should not crash, no matter how the non-determinism is resolved:

$$
\text { type-soundness: } \begin{aligned}
& {[] \vdash t \in \sigma \rightarrow } \\
&\neg \text { (now nothing } \approx \in \llbracket t \rrbracket[])
\end{aligned}
$$

It is easy to prove this statement by adapting the proof from Section 4. All it takes is to extend the Lift type with the constructor

$$
\text { - }_{-}: \text {Lift } P x \rightarrow \text { Lift } P y \rightarrow \text { Lift } P(x \mid y),
$$

and then propagating this change through the rest of the proof. Note that the new definition of Lift uses induction nested inside coinduction (as do $D$ and $\_\approx_{-}^{\epsilon}$ ).

\section{Term Equivalences}

Let us now return to the deterministic language from Section 3. Weak bisimilarity as defined in Section 2 is, despite its name, a very strong notion of equality for the semantic domain (Maybe Value $)_{\perp}$. We can lift this equality to closed terms in the following way:

$$
\begin{aligned}
& { }_{-}: \operatorname{Tm} 0 \rightarrow \operatorname{Tm} 0 \rightarrow \operatorname{Set} \\
& t_{1} \equiv t_{2}=\llbracket t_{1} \rrbracket[] \approx \llbracket t_{2} \rrbracket[]
\end{aligned}
$$

This is a very syntactic equality, which distinguishes the observationally equivalent terms $t_{1}=\operatorname{lam}(\operatorname{lam}(\operatorname{var} 0)) \cdot \operatorname{con} 0$ and $t_{2}=$ lam (var 0$)$, because

$$
\begin{aligned}
& \text { 【t } t_{1} \rrbracket[] \quad \approx \\
& \text { return }(\operatorname{lam}(\operatorname{var} 0)(\operatorname{con} 0::[])) \not \\
& \text { return }(\operatorname{lam}(\operatorname{var} 0) \quad[]) \approx \\
& \llbracket t_{2} \rrbracket[] \text {. }
\end{aligned}
$$

The relational big-step semantics from Section 5 is no different: []$\vdash t_{1} \Downarrow v$ does not imply that we have [] $\vdash t_{2} \Downarrow v$.

This section defines some less syntactical term equivalences. Discussion of the finer points of these equivalences is out of scope for this paper; the main point is that they can be defined without too much fuss. 
Let us start by defining a notion of applicative bisimilarity (Abramsky 1990). Computations are equivalent $\left({ }_{-} \approx_{\perp_{-}}\right)$if they are weakly bisimilar, with equivalent (rather than equal) possibly exceptional values; possibly exceptional values are equivalent $\left({ }_{-} \approx_{\mathrm{MV}-}\right)$ if they are of the same kind and, in the case of success, contain equivalent values; and values are equivalent $\left({ }_{-} \approx_{V_{-}}\right)$if they are either equal constants, or closures which are equivalent when evaluated with the free variables bound to an arbitrary value: ${ }^{7}$

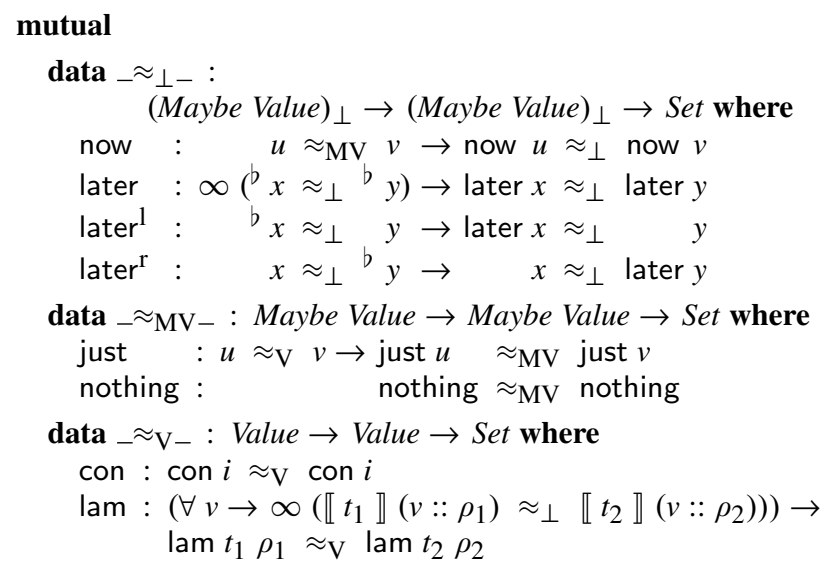

This is yet again a definition which uses induction nested inside coinduction. Note that the lam constructor is coinductive. If this constructor were inductive, then the relations would not be reflexive: lam (var zero) [] would be provably distinct from itself.

Using the relations above we can define applicative bisimilarity by stating that terms are equivalent if they are equivalent when evaluated in an arbitrary context:

$$
\begin{aligned}
& \approx_{\mathrm{T}-}: \operatorname{Tm} n \rightarrow \operatorname{Tm} n \rightarrow \text { Set } \\
& t_{1} \approx_{\mathrm{T}} t_{2}=\forall \rho \rightarrow \llbracket t_{1} \rrbracket \rho \approx_{\perp} \llbracket t_{2} \rrbracket \rho
\end{aligned}
$$

The definition of $\_\approx_{\perp-}$ is very similar to the definition of weak bisimilarity in Section 2. It is possible to define a single notion of weak bisimilarity, parametrised by a relation to use for values. The accompanying code uses such a definition.

Let us now turn to contextual equivalence. Contexts with zero or more holes can be defined as follows:

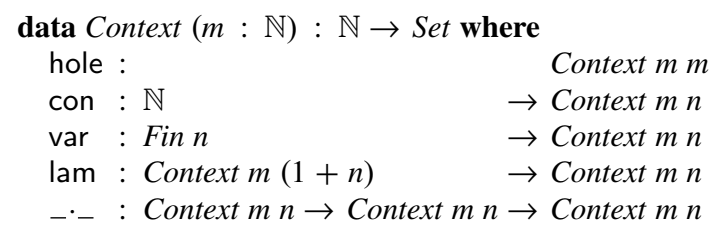

The type Context $m n$ contains contexts whose holes expect terms of type $T m m$. If we fill the holes, then we get a term of type $T m n$ :

$$
\begin{array}{lrl}
\text { ___ }]: & \text { Context } m \rightarrow T m \rightarrow T m n \\
\text { hole } & {[t]=t} \\
\text { con } i & {[t]=\operatorname{Tm}=\operatorname{con} i} \\
\operatorname{var} x & {[t]=\operatorname{var} x} \\
\operatorname{lam} C & {[t]=\operatorname{lam}(C[t])} \\
\left(C_{1} \cdot C_{2}\right)[t]=C_{1}[t] \cdot C_{2}[t]
\end{array}
$$

Contextual equivalence can be defined in two equivalent ways. The usual one states that $t_{1}$ and $t_{2}$ are contextually equivalent if $C\left[t_{1}\right]$ terminates iff $C\left[t_{2}\right]$ terminates, for any closing context $C$ :

$$
\begin{aligned}
& -\Downarrow: A_{\perp} \rightarrow \text { Set } \\
& x \Downarrow=\exists \lambda v \rightarrow x \approx \text { now } v
\end{aligned}
$$

\footnotetext{
$\overline{7} \forall v \rightarrow \ldots$ means the same as $\left(v:{ }_{-}\right) \rightarrow \ldots$; Agda tries to infer the value
} of the underscore automatically.

$$
\begin{aligned}
& -\approx_{\mathrm{C}-}: T m n \rightarrow T m n \rightarrow S e t \\
& t_{1} \approx_{\mathrm{C}} t_{2}=\forall C \rightarrow \llbracket C\left[t_{1}\right] \rrbracket[] \Downarrow \Leftrightarrow \mathbb{T} \Leftrightarrow\left[t_{2}\right] \rrbracket[] \Downarrow
\end{aligned}
$$

However, we can also define contextual equivalence using weak bisimilarity:

$$
\begin{aligned}
& -\approx_{\mathrm{C}^{-}}^{\prime}: \operatorname{Tm} n \rightarrow T m n \rightarrow \text { Set } \\
& t_{1} \approx_{\mathrm{C}}^{\prime} t_{2}=\forall C \rightarrow \llbracket C\left[t_{1}\right] \rrbracket[] \approx^{\circ} \llbracket C\left[t_{2}\right] \rrbracket[]
\end{aligned}
$$

Here $\approx_{-}^{\circ}$ is a notion of weak bisimilarity which identifies all terminating computations:

$$
\text { now : now } u \approx^{\circ} \text { now } v
$$

It is easy to prove that these two notions of contextual equivalence are equivalent.

As an aside one can note that the contextual equivalences above are a bit strange, because there is no context which distinguishes con 0 from con 1 . This could be fixed by extending the language with suitable constructions for observing the difference between distinct constants.

\section{Conclusions}

When writing down a semantics I think one of the main priorities should be to make it easy to understand. Sometimes a more complicated definition may be more convenient for certain tasks, but in that case one can define two semantics and prove that they are equivalent.

I hope I have convinced you that functional operational semantics defined using the partiality monad are easy to understand. I have also used two such semantics to state a compiler correctness result, and I find this statement to be easier to understand than a corresponding statement phrased using relational semantics (see Section 7).

The semantics also seem to be useful when it comes to proving typical meta-theoretic properties, at least for the simple languages discussed in this paper. I have proved type soundness and compiler correctness directly for the semantics given above. The type soundness proof in Section 4 is given in relatively complete, formal detail, yet it is short and should be easy to follow. Furthermore, as mentioned in Section 7, the compiler correctness proof avoids some duplication which is present in a corresponding proof for relational semantics.

As discussed above the support for total corecursion in languages like Agda and Coq is somewhat limited: definitions like 【-】are often rejected. However, my experience with sized types in MiniAgda (see Section 2) is encouraging. I suspect that a more polished implementation of sized types could be quite satisfying to work with.

Finally I want to mention a drawback of this kind of semantics: proofs which proceed by induction on the structure of $\_\vdash_{-} \Downarrow_{-}$ when a relational big-step semantics is used can become somewhat awkward when transferred to this setting, as illustrated by the proof in Section 5 showing that $\llbracket t \rrbracket \rho \approx$ return $v$ implies $\rho \vdash t \Downarrow v$. However, it is unclear to me how often this is actually a problem. For instance, neither the type soundness proofs nor the compiler correctness proofs in this paper are affected by this drawback.

\section{Acknowledgements}

I want to thank Thorsten Altenkirch for encouraging this line of work, Peter Dybjer for useful feedback on a draft of the paper, and Tarmo Uustalu for pointing out some related work. I would also like to thank the anonymous reviewers for lots of useful feedback.

Large parts of this work were done when I was working at the University of Nottingham, with financial support from EPSRC (grant code: EP/E04350X/1). I have also received support from the 
ERC: "The research leading to these results has received funding from the European Research Council under the European Union's Seventh Framework Programme (FP7/2007-2013) / ERC grant agreement n ${ }^{\circ} 247219$."

\section{References}

Andreas Abel. MiniAgda: Integrating sized and dependent types. In Proceedings Workshop on Partiality and Recursion in Interactive Theorem Provers (PAR 2010), volume 43 of EPTCS, 2010. doi:10.4204/EPTCS. 43.2 .

Samson Abramsky. The lazy lambda calculus. In Research Topics in Functional Programming. Addison-Wesley, 1990.

The Agda Team. The Agda Wiki. Available at http://wiki.portal. chalmers.se/agda/, 2012.

Thorsten Altenkirch and Nils Anders Danielsson. Termination checking in the presence of nested inductive and coinductive types. Short note supporting a talk given at the Workshop on Partiality and Recursion in Interactive Theorem Provers (PAR 2010), 2010.

Brian E. Aydemir, Aaron Bohannon, Matthew Fairbairn, J. Nathan Foster, Benjamin C. Pierce, Peter Sewell, Dimitrios Vytiniotis, Geoffrey Washburn, Stephanie Weirich, and Steve Zdancewic. Mechanized metatheory for the masses: The PoplMark challenge. In Theorem Proving in Higher Order Logics, 18th International Conference, TPHOLs 2005, volume 3603 of $L N C S$, pages 50-65, 2005. doi:10.1007/11541868_4.

Nick Benton and Chung-Kil Hur. Biorthogonality, step-indexing and compiler correctness. In ICFP'09, Proceedings of the 2009 ACM SIGPLAN International Conference on Functional Programming, pages 97-107, 2009. doi:10.1145/1596550.1596567.

Nick Benton, Andrew Kennedy, and Carsten Varming. Some domain theory and denotational semantics in Coq. In Theorem Proving in Higher Order Logics, 22nd International Conference, TPHOLs 2009, volume 5674 of LNCS, pages 115-130, 2009. doi:10.1007/978-3-642-03359-9_10.

Venanzio Capretta. General recursion via coinductive types. Logical Methods in Computer Science, 1(2):1-28, 2005. doi:10.2168/LMCS-1(2: 1)2005.

The Coq Development Team. The Coq Proof Assistant, Reference Manual, Version 8.3pl3, 2011.

Thierry Coquand. Infinite objects in type theory. In Types for Proofs and Programs, International Workshop TYPES '93, volume 806 of LNCS, pages 62-78, 1994. doi:10.1007/3-540-58085-9_72.

Patrick Cousot and Radhia Cousot. Inductive definitions, semantics and abstract interpretations. In POPL '92, Proceedings of the 19th ACM SIGPLAN-SIGACT symposium on Principles of programming languages, pages 83-94, 1992. doi:10.1145/143165.143184.

Patrick Cousot and Radhia Cousot. Bi-inductive structural semantics. Information and Computation, 207(2):258-283, 2009. doi:10.1016/j.ic.2008. 03.025.

Nils Anders Danielsson. Beating the productivity checker using embedded languages. In Proceedings Workshop on Partiality and Recursion in Interactive Theorem Provers (PAR 2010), volume 43 of EPTCS, pages 29-48, 2010. doi:10.4204/EPTCS.43.3.

Nils Anders Danielsson and Thorsten Altenkirch. Subtyping, declaratively: An exercise in mixed induction and coinduction. In Mathematics of Program Construction, 10th International Conference, MPC 2010, volume 6120 of $L N C S$, pages 100-118, 2010. doi:10.1007/978-3-642-13321-3_ 8.

Neil Ghani and Tarmo Uustalu. Monad combinators, non-determinism and probabilistic choice. Extended abstract distributed at the workshop on Categorical Methods in Concurrency, Interaction and Mobility (CMCIM 2004), 2004.

Sergey Goncharov and Lutz Schröder. A coinductive calculus for asynchronous side-effecting processes. In Fundamentals of Computation Theory, 18th International Symposium, FCT 2011, volume 6914 of LNCS, pages 276-287, 2011. doi:10.1007/978-3-642-22953-4_24.

Graham Hutton. Programming in Haskell. Cambridge University Press, 2007.
Xavier Leroy. Formal verification of a realistic compiler. Communications of the ACM, 52:107-115, 2009. doi:10.1145/1538788.1538814.

Xavier Leroy and Hervé Grall. Coinductive big-step operational semantics. Information and Computation, 207(2):284-304, 2009. doi:10.1016/j.ic. 2007.12.004

Robin Milner and Mads Tofte. Co-induction in relational semantics. Theoretical Computer Science, 87(1):209-220, 1991. doi:10.1016/ 0304-3975(91)90033-X.

Eugenio Moggi. An abstract view of programming languages. Technical Report ECS-LFCS-90-113, Lab. for Found. of Comp. Sci., University of Edinburgh, 1990.

Eugenio Moggi. Notions of computation and monads. Information and Computation, 93(1):55-92, 1991. doi:10.1016/0890-5401(91)90052-4.

Keiko Nakata and Tarmo Uustalu. Trace-based coinductive operational semantics for While: Big-step and small-step, relational and functional styles. In Theorem Proving in Higher Order Logics, 22nd International Conference, TPHOLs 2009, volume 5674 of LNCS, pages 375-390, 2009. doi:10.1007/978-3-642-03359-9_26.

Keiko Nakata and Tarmo Uustalu. Resumptions, weak bisimilarity and big-step semantics for While with interactive I/O: An exercise in mixed induction-coinduction. In Proceedings Seventh Workshop on Structural Operational Semantics (SOS 2010), volume 32 of EPTCS, pages 57-75, 2010. doi:10.4204/EPTCS.32.5.

Ulf Norell. Towards a practical programming language based on dependent type theory. PhD thesis, Chalmers University of Technology and Göteborg University, 2007.

Christine Paulin-Mohring. A constructive denotational semantics for Kahn networks in Coq. In From Semantics to Computer Science: Essays in Honour of Gilles Kahn, pages 383-413. Cambridge University Press, 2009.

John C. Reynolds. Definitional interpreters for higher-order programming languages. In $A C M$ ' 72 , Proceedings of the ACM annual conference, volume 2, pages 717-740, 1972. doi:10.1145/800194.805852.

J.J.M.M. Rutten. A note on coinduction and weak bisimilarity for while programs. Theoretical Informatics and Applications, 33:393-400, 1999. doi:10.1051/ita:1999125.

Davide Sangiorgi and Robin Milner. The problem of "weak bisimulation up to". In CONCUR '92, Third International Conference on Concurrency Theory, volume 630 of $L N C S$, pages 32-46, 1992. doi:10.1007/ $\mathrm{BFb} 0084781$.

Mads Tofte. Type inference for polymorphic references. Information and Computation, 89(1):1-34, 1990. doi:10.1016/0890-5401(90)90018-D.

Philip Wadler. The essence of functional programming. In $P O P L$ '92, Proceedings of the 19th ACM SIGPLAN-SIGACT symposium on Principles of programming languages, pages 1-14, 1992. doi:10.1145/143165. 143169. 